\title{
Pyogenic Granuloma of Nasal Septum
}

\author{
${ }^{1}$ Anuja Santosh Kulkarni, ${ }^{2}$ Bachi T Hathiram \\ ${ }^{1}$ Assistant Divisional Medical Officer and Consultant, Department of ENT and Head and Neck Surgery, Jagjivan Ram Hospital \\ Mumbai, Maharashtra, India \\ ${ }^{2}$ Professor and Head, Department of ENT and Head and Neck Surgery, BYL Nair Charitable Hospital and Topiwala National Medical College \\ Mumbai, Maharashtra, India
}

Correspondence: Anuja Santosh Kulkarni, Assistant Divisional Medical Officer and Consultant, c/o Santosh J Kulkarni, H. No. 809 Shri Yashwant Maharaj Mandir, Nashik-400001, Maharashtra, India, Phone: 9004490547, e-mail: dr.anujakulkarni@gmail.com

\section{ABSTRACT}

Pyogenic granuloma also known as lobular capillary hemangioma is although rare but well-documented clinical entity. However, it is more commonly reported in cases pertaining to lesions of oral cavity and especially in female patients during pregnancy usually in third decade of life. But, here we report a very uncommon presentation of pyogenic granuloma arising from nasal septum in a 52-year-old male patient who presented to us with history of epistaxis and left-sided nasal obstruction since 4 months.

Keywords: Pyogenic granuloma, Lobular capillary hemangioma, Epistaxis.

\section{CASE REPORT}

A 52-year-old male patient came with chief complaints of history of epistaxis from left nostril since last 4 months which was spontaneous in onset, moderate in amount, four to five episodes per week stopped on conservative treatment. This was associated with left-sided nasal blockage. There was also history of mucoid, bloodstained discharge from the left nostril as well as history of anosmia. There was neither history suggestive of atrophic rhinitis nor that of sinusitis. There was neither any history suggestive of bleeding tendency nor that of jaundice. There was no history of prolonged used of painkillers or any antiplatelet drugs in the past. There was no history of major medical or surgical illness in past.

On examination of nose, there was no external nasal deformity. There was deviation of nasal septum toward right. There was left middle turbinate hypertrophy. A pinkish polypoidal pedunculated mass was present attached to posterior aspect of nasal septum, insensitive to touch and did not bleed on touch. Air blast was reduced on left side. There was no paranasal sinus tenderness. On posterior rhinoscopy, there was no abnormality detected. Ear and throat examination did not reveal any abnormality.

Patient was investigated subsequently. Diagnostic nasal endoscopy performed with zero degree endoscope which revealed $2.4 \times 0.5 \times 2.5 \mathrm{~cm}$ sized pinkish, polypoidal, fleshy, pedunculated mass attached to posterior aspect of nasal septum; extended posteriorly up to nasopharynx; insensitive to and did not bleed on touch (Fig. 1). Endoscopic excision of mass with cauterization of base done; hemostasis achieved. Mass was sent for histopathology and immunohistochemistry.

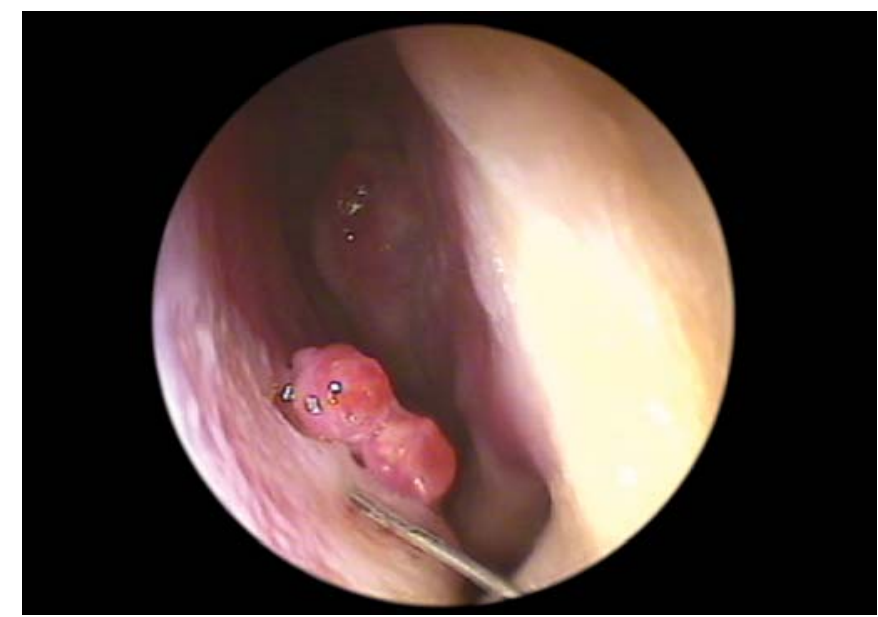

Fig. 1: Endoscopic appearance of pyogenic granuloma

Histology revealed lobular mass closed by stratified squamous epithelium with focal bridging and ulceration. Stroma showed fibroconnective tissue with plenty of capillaries lined by flattened endothelial cells containing RBCs. This was suggestive of lobular capillary hemangioma also called as pyogenic granuloma (Figs 2 and 3).

On immunohistochemistry, the tissue was positive for CD31 and CD34 markers both highlighting the endothelial cell linings suggesting strong angiogenetic potential of mass (Figs 4 and 5).

\section{DISCUSSION}

Pyogenic granuloma arising from the nasal septum is a very rare clinical entity. ${ }^{1}$ Pyogenic granuloma also known as lobular capillary hemangioma is more commonly reported entity in cases of oral cavity lesions. It is predominantly 


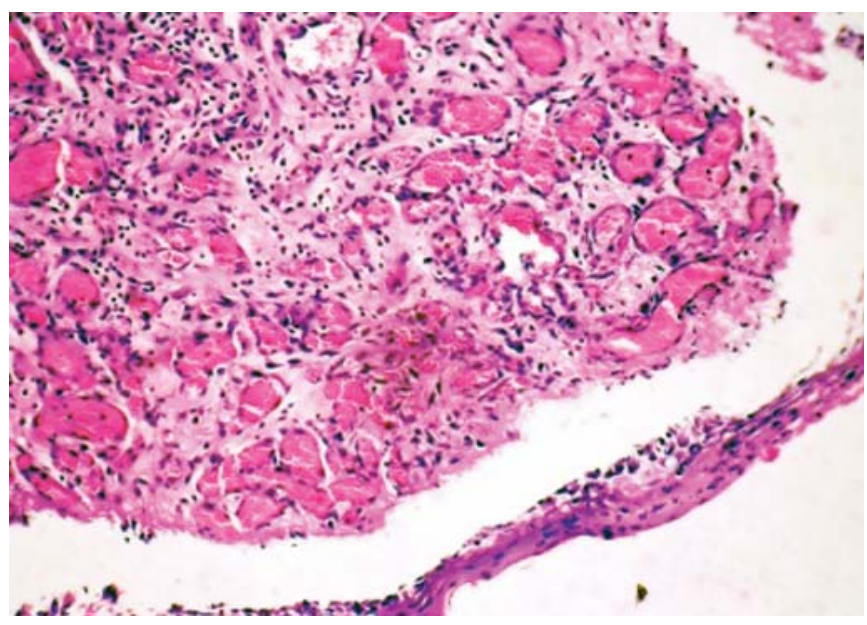

Fig. 2: Photomicrograph of histological appearance of pyogenic granuloma (lobular capillary hemangioma) of nasal septum $\mathrm{H}$ \& $\mathrm{E}$ staining (magnification 10x)

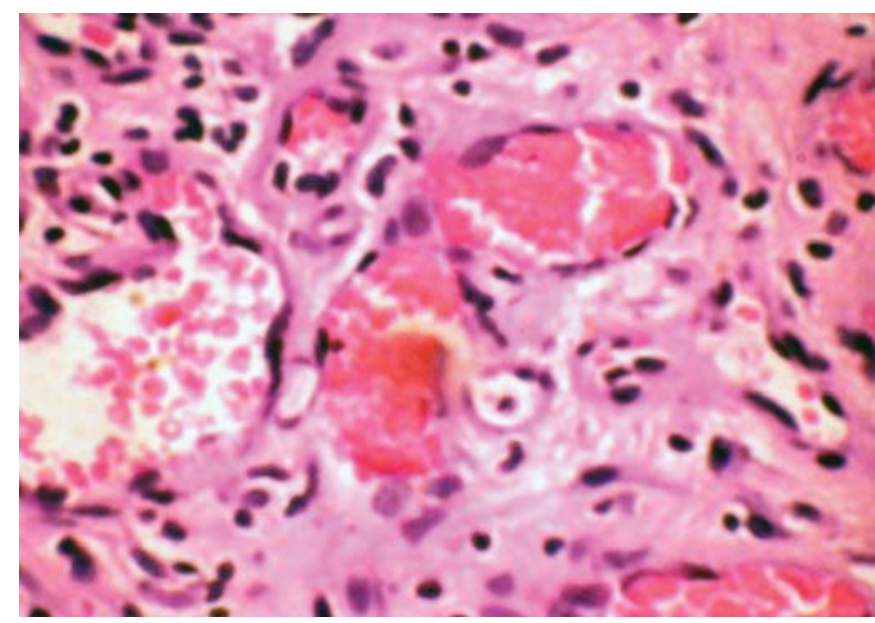

Fig. 3: Histological appearance of pyogenic granuloma of nasal septum on higher magnification $\mathrm{H}$ \& E staining (magnification $40 \mathrm{x}$ )

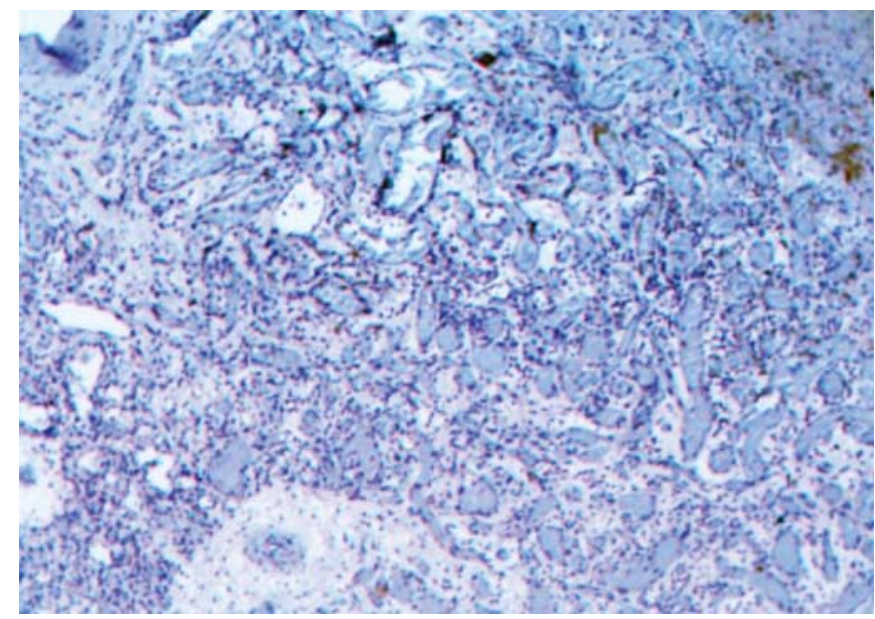

Fig. 4: Immunohistochemistry showing lesion positive for CD31 marker highlighting the endothelial linings

seen in female patients, usually in their third decade of life, especially during pregnancy; ${ }^{2-4}$ hence, it is also known as pyogenic granuloma gravidarum.

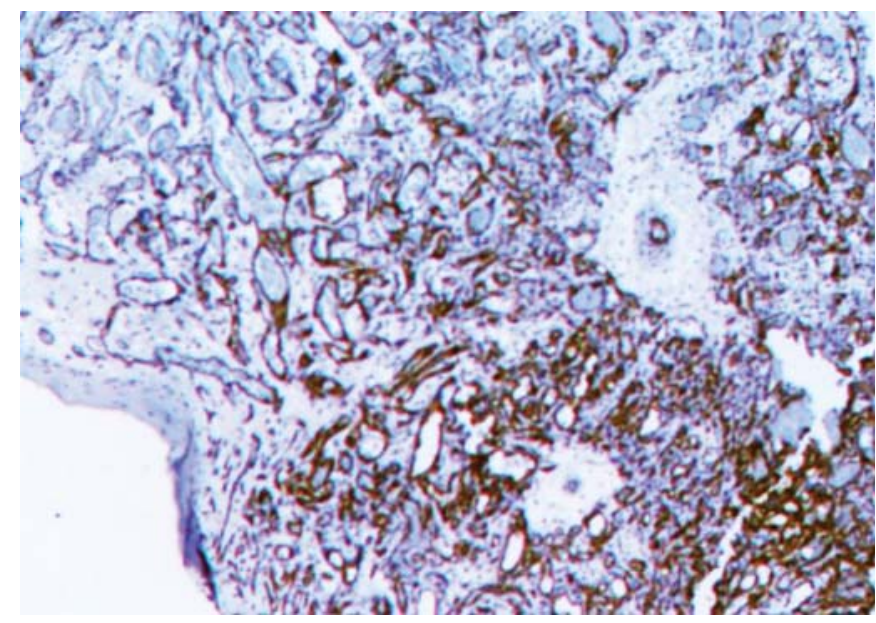

Fig. 5: Immunohistochemistry study showing lesion strongly positive for CD34 marker highlighting endothelial cell linings

Pyogenic granuloma is a misnomer as previously this entity was thought to be caused due to bacterial infection but it is not so. It is neither a true neoplasm too. It is caused due to abnormal proliferation of capillaries in lobular pattern. ${ }^{3-5}$ It is also one of the mimickers of Kaposis sarcoma. Immunostaining for human herpesvirus 8 latent nuclear antigen-1 helps to distinguish Kaposi sarcoma from its mimickers. ${ }^{6}$ Among the various immunochemical markers tried, this lesion is consistently positive for CD31 and CD34 markers as these markers selectively highlight vascular endothelial cells. ${ }^{6-8}$ Local trauma or trauma due to intubation is considered as one of the etiological factors. ${ }^{9}$ Presence of pyogenic granuloma causes recurrent unilateral epistaxix and nasal obstruction. Differential diagnosis includes hamartomas, venous hemangioma, arteriovenous malformation, nasal polyp.

Conclusively, pyogenic granuloma should be considered as one of the important clinical entity as a differential diagnosis in a patient presenting with history of recurrent nasal bleed.

\section{REFERENCES}

1. Yousry El-Sayeda1 CL, Al-Serhania Awad. Lobular capillary haemangioma (pyogenic granuloma) of the nose. Journal of Laryngology \& Otology 1997;111:941-45.

2. Cengiz Ozcan, Düsmez Apa Duygu, Kemal Görür. Pediatric lobular capillary hemangioma of the nasal cavity. Eur Arch Otorhinolaryngol 2004;261(8):449-51.Epub 2003.

3. Yuan K, Wing LY, Lin MT. Pathogenetic roles of angiogenic factors in pyogenic granulomas in pregnancy are modulated by female sex hormones. J Periodontol 2002;73(7):701-08.

4. Sampurna Roy. Pathological case of the month: Diagnosis and discussion; pyogenic granuloma of the tongue. Arch Pediatr Adolesc Med 2001;155,1065-66.

5. Nair Lt Col S, Bahal Maj A, Bhadauria Col RS. Lobular capillary haemangioma of the nasal cavity: Observation of three specific cases. Acta Otorhinolaryngol Belg 2001;55(3):241-46.

6. Cheuk W, Wong KO, Wong CS, Dinkel JE, Ben-Dor D. Immunostaining for human herpesvirus \& latent nuclear antigen-1 
helps distinguish kaposi sarcoma from its mimickers. Am J Clin Pathol 2004;121(3):335-42.

7. Yuan K, Jin YT, Lin MT. The detection and comparison of angiogenesis-associated factors in pyogenic granuloma by immunohistochemistry. J Periodontol 2000;71(5):701-09.

8. Epivatianos A, Antoniades D, Zaraboukas T, Zairi E, Poulopoulos A, Kiziridou A, et al. Pyogenic granuloma of the oral cavity: Comparative study of its clinicopathological and immunohistochemical features. Pathol Int 2005;55(7): 391-97.

9. Neves-Pinto RM, Carvalho A, Araujo E, Alberto C, BasilioDe-Oliveira GA. Nasal septum giant pyogenic granuloma after a long lasting nasal intubation: Case report. Rhinology Mar 2005; 43(1):66-69. 